\title{
Intravenous immunoglobulin therapy among pediatric patients: labeled and off-labeled indications
}

\author{
Chin Chiang Toh¹, Farida Islahudin', Adli Ali², Noraida Mohamed Shah",** \\ ${ }^{1}$ Faculty of Pharmacy, Universiti Kebangsaan Malaysia, Kuala Lumpur, ${ }^{2}$ Pediatric Department, Faculty of Medicine, \\ Universiti Kebangsaan Malaysia Medical Centre, Kuala Lumpur
}

\begin{abstract}
This study was designed to evaluate utilization patterns and clinical outcome of intravenous immunoglobulin (IVIG) therapy among pediatric patients in a tertiary hospital. Demographic data, IVIG prescribed, and clinical outcome were retrospectively reviewed from the pharmacy dispensing data and patient medical records between 2007 and 2014. One hundred and fifteen instances of IVIG administration to 108 pediatric patients were recorded. A total of 61 cases $(53 \%)$ and 54 cases (47\%) of the IVIG administered were for labeled and off-labeled indications, respectively. Age, weight, specialty, total IVIG usage, length of hospital stays, and mortality rate were found to be significantly associated with the indication being labeled or off-labeled $(p<0.05)$. However, there was no significant difference in terms of adverse reactions between labeled and off-labeled indications $(\mathrm{p}>0.05)$. Guidelines should be developed and implemented for rational and evidence-based use of IVIG to avoid unnecessary wastage.
\end{abstract}

Keywords: Intravenous Immunoglobulin. Off-label indications. Pediatric patients. Malaysia hospital

\section{INTRODUCTION}

Over the past two and a half decades, the administration of intravenous immunoglobulin (IVIG) has become an important therapy in clinical medicine. These preparations, which contain a broad range of antibody specificities, were originally used in antibody replacement therapy. However, many other clinical benefits of IVIG treatment have been demonstrated (Orange et al., 2006). A rapid increase in the use of IVIG for an ever-growing number of conditions in children and neonates was also reported (Stiehm, Keller, Vyas, 2008).

In pediatrics, IVIG has been found to have a major impact in the treatment of conditions in the fields of neurology, hematology, rheumatology, dermatology, neonatology, immunology, cardiology, and infectious diseases (Prasad, Chaudhary, 2014). More than 150 offlabeled indications of IVIG in 128 clinical trials and 268 case reports were reported in another literature review by Leong et al. (2008). The most common off-labeled indications include multiple sclerosis, prevention of

\footnotetext{
*Correspondence: N. Mohamed Shah. Faculty of Pharmacy, Universiti Kebangsaan Malaysia, Jalan Raja Muda Abdul Aziz, 50300 Kuala Lumpur. E-mail: noraida_mohamedshah@yahoo.com. Phone: +60392898038. Fax: $+60392893271$
}

antiphospholipid syndrome in miscarriage, Guillain-Barré syndrome, and progression of human immunodeficiency virus (HIV) after delivery. Health care institutions are strongly urged to closely monitor specific uses of IVIG to reserve supplies for the "best-evidence" uses because of the limited knowledge associated with off-labeled indications (Leong et al., 2008).

IVIG is known to be a complex therapy with adverse effects. Adverse reactions associated with the regular administration of IVIG still occur despite the introduction of manufacturing steps that enhance purity, minimize damage to the Immunoglobulin G molecules, and result in a higher concentration of liquid IVIG (Bichuetti-Silva et al., 2014). In 2002, a survey of more than 1000 patients with primary immunodeficiency conducted by the Immune Deficiency Foundation (IDF) found that $44 \%$ of the reported adverse reactions were not related to the rate of infusion but to the drug itself. Systemic reactions to IVIG infusion (not related to the rate of infusion) were reported to range from $3 \%$ to $15 \%$, which include backache, abdominal pain, nausea, chills, rhinitis, asthma, low-grade fever, myalgia, and headaches (Goddard, 2008).

This study was designed to evaluate utilization patterns and clinical outcome of the IVIG therapy in a 
tertiary teaching hospital in Malaysia focusing on the offlabel indications.

\section{MATERIAL AND METHODS}

This observational retrospective chart review study was approved by the Research Ethics Committee of UKMMC (UKM 1.5.3.5/244/NF-009-15). Data collection was carried out from April to June 2015. Pediatric patients ranging in age from birth to 12 years who were administered IVIG from January 2007 to December 2014 during their hospital stay were traced using the IVIG usage logbook at the pharmacy department of UKMMC. Medical records were then retrieved from the Medical Record Unit and screened for confirmation of IVIG administration. Detailed clinical data including demographic data, primary diagnosis, dosing regimen of IVIG, indication, and clinical outcome were recorded using a designated data collection form.

The indication of IVIG is mainly divided into two categories, labeled and off-labeled. Labeled indication refers to the seven indications approved by the FDA as follows:

a. Treatment of primary immunodeficiency

b. Prevention of bacterial infections in patients with hypogammaglobulinemia and recurrent infection caused by B-cell chronic lymphocytic leukemia

c. Prevention of coronary artery aneurysms in Kawasaki disease

d. Prevention of infections, pneumonia, and acute graft versus host disease (GVHD) after bone marrow transplantation

e. Reduction of serious bacterial infection in children with HIV

f. Increase of platelet count in idiopathic thrombocytopenic purpura (ITP) to prevent or control bleeding

g. Chronic inflammatory demyelinating polyneuropathy (CIDP)

Appropriate use of IVIG was placed into one of three a priori agreed upon categories. Off-labeled indication is defined as the indication of IVIG other than the seven indications approved by the FDA (Prasad, Chaudhary, 2014). Off-labeled indication is further categorized into 2 subgroups, "potentially indicated" or "not indicated." "Off-labeled, potentially indicated" is the term used for disorders with clinical information supporting the use of IVIG as listed in the British Columbia IVIG Utilization Management Handbook and/or in the panel recommendations of the Canadian Blood Service 2000 national consensus conference. Clinical indications with no convincing evidence of benefit were defined as "not indicated." These were used for conditions with no evidence supporting the use of IVIG or for conditions in which IVIG use was deemed ineffective (Selin, 2002; Sher et al., 2000). The level of evidence for the labeled and offlabeled indication is classified as category I, IIa, IIb, or III (Sher et al., 2000).

As clinical outcomes, three parameters were documented: the length of hospital stay, mortality, and adverse reaction. Length of hospital stay is defined as the duration of hospital stay from the day of hospital admission until the patient is discharged or deceased. Mortality is defined as all-cause mortality. Adverse reaction related to IVIG infusion refers to a reaction that occurred during or within 48 hours after the infusion. It can be classified as mild, moderate, or severe as below (Brennan et al., 2003):

a. Mild reactions

Symptoms included headache, flushing, muscle aches, shivering, feeling sick, itching, urticaria, anxiety, lightheadedness, dizziness, or irritability. These subsided when the infusion rate was decreased.

b. Moderate reactions

Symptoms included mild reactions becoming worse or other symptoms such as chest pain or wheezing, necessitating the infusion to be discontinued.

c. Severe reactions

Symptoms included moderate reactions persisting or becoming worse, or other symptoms such as tightness of the throat, severe headache and shaking, severe breathlessness or wheezing, severe dizziness or fainting, sensation of pressure in the chest, or collapsing. A severe reaction would require the administration of adrenaline and medical attention. Statistical Package for the Social Sciences version 22 (SPSS ${ }^{\circledR}$, Chicago, IL) was used to perform all statistical analyses. The indication, dosing regimen, and outcome of IVIG administration were descriptively analyzed. The Chi Square and Fisher's Exact tests were used to compare two categorical variables, and the Mann-Whitney U test was used in comparing the numerical and categorical variables. All statistical tests were two-tailed. A p-value of $<0.05$ denoted statistical significance.

\section{RESULTS}

A total of 162 patients with prescribed IVIG were identified through the computerized pharmacy system with only 118 patients having traceable medical records. The remaining 44 medical records were untraceable due to patients defaulting follow up for 5 years or more. Of the 118 patients, only 3 did not receive the IVIG therapy due to the patients' worsening clinical condition. Hence, the 
final sample size was 115 instances of IVIG administered to 108 patients from January 1, 2007 to December 31, 2014. The patient characteristics and clinical data are summarized in Table I.

Out of the 115 cases of IVIG administered, 61 cases $(53 \%)$ were used for FDA approved indications while another 54 cases (470\%) were used for off-labeled indications. The indications for IVIG had a category I evidence level in $68.9 \%$ of cases whereas the remaining $39.1 \%$ had category II and III evidence level (Table II).

Over the 8-year period, the total IVIG usage was $1396.42 \mathrm{~g}$. A total of $1007.70 \mathrm{~g}$ and $388.72 \mathrm{~g}$ of IVIG were administered for labeled and off-labeled indications, respectively. The dose per course ranged from $0.4 \mathrm{mg} / \mathrm{kg}$ to $2.0 \mathrm{mg} / \mathrm{kg}$ with a median of $1.5 \mathrm{mg} / \mathrm{kg}$. Sixty-nine percent of IVIG cases $(n=79)$ were administered in a single dose and the remaining $31 \%(n=36)$ were administered as divided doses, up to 5 doses, with an interval of 2 to 5 days per case.

The length of hospital stays for a patient who received the IVIG ranged from 1 to 361 days, with a median of 23.8 days. After IVIG administration, 99 patients (86.1\%) were discharged. Sixteen (13.9\%) patients died but death was not related to IVIG administration. The cause of death comprised 15 cases of severe neonatal sepsis with underlying prematurity and 1 case of severe gastrointestinal bleeding.

Seven adverse reactions related to the IVIG administration were identified: 5 mild cases and 1 case each of moderate and severe adverse reactions. Patients with mild adverse reactions developed fever during the IVIG administration, and the symptom subsided when the infusion rate was reduced. Moderate adverse reaction was seen in patients who presented with chills, rigors, fever, mild bronchospasm, mild tachypnea, and tachycardia, which required additional intervention with antipyretic, bronchodilator, oxygen and necessitating the termination of IVIG infusion. In the severe adverse reaction case, the patient presented with general tonic-clonic seizure during IVIG infusion and required a loading dose of an antiepileptic drug, intubation for airway protection, and termination of IVIG infusion. The Naranjo scale was used to determine the probability of IVIG-related adverse reactions for the 7 cases, and the scores ranged from 5 to 8 .

In this study, the patient's age, body weight, total usage of IVIG, specialty, length of hospital stay, and allcause mortality were found to be significantly associated with the indication being labeled or off-labeled $(\mathrm{p}<0.001)$. There were no significant association between the indication of IVIG with adverse reaction related to IVIG infusion, gender and ethnicity ( $\mathrm{p}>0.05)$ (Table III).
TABLE I - Patient demographics and clinical data $(n=108$ patients)

\begin{tabular}{|c|c|}
\hline Parameter & $\mathbf{n}$ \\
\hline$\overline{\text { Courses of IVIG }(n)}$ & 115 \\
\hline Age, years [median (IQR)] & $0.58(1.98)$ \\
\hline \multicolumn{2}{|l|}{ Gender, $n(\%)$} \\
\hline Male & $65(56.5)$ \\
\hline Female & $50(43.5$ \\
\hline Body weight, kg [median (IQR)] & $6.88(8.76)$ \\
\hline \multicolumn{2}{|l|}{ Ethnicity, $n(\%)$} \\
\hline Malay & $75(65.2)$ \\
\hline Chinese & $33(28.7)$ \\
\hline Indian & $2(1.7)$ \\
\hline Others & $5(4.3)$ \\
\hline \multicolumn{2}{|l|}{ Setting, $n(\%)$} \\
\hline PHDU & $69(60.0)$ \\
\hline NICU & $44(38.3)$ \\
\hline PICU & $2(1.7)$ \\
\hline \multicolumn{2}{|l|}{ Specialty, n (\%) } \\
\hline Neurology & $7(6.1)$ \\
\hline Hematology & $31(27.0)$ \\
\hline Immunology & $4(3.4)$ \\
\hline Dermatology & $25(21.7)$ \\
\hline Neonatology & $44(38.3)$ \\
\hline Others & $4(3.4)$ \\
\hline \multicolumn{2}{|l|}{ Primary diagnosis, $n(\%)$} \\
\hline ITP & $31(27.0)$ \\
\hline Neonatal sepsis & $31(27.0)$ \\
\hline Kawasaki disease & $24(20.9)$ \\
\hline $\begin{array}{l}\text { Severe neonatal jaundice (NNJ) secondary } \\
\text { ABO incompatibility }\end{array}$ & $8(7.0)$ \\
\hline Primary immunodeficiency disease & $3(2.6)$ \\
\hline Guillain-Barré syndrome & $2(1.7)$ \\
\hline $\begin{array}{l}\text { Perinatal acquired varicella infection } \\
\text { (prophylaxis) }\end{array}$ & $2(1.7)$ \\
\hline Neonatal autoimmune thrombocytopenia & $2(1.7)$ \\
\hline CIDP & $2(1.7)$ \\
\hline Multiple sclerosis & $1(0.9)$ \\
\hline Acute graft versus host disease (GVHD) & $1(0.9)$ \\
\hline Varicella pneumonitis & $1(0.9)$ \\
\hline Anti-NMDA receptor encephalitis & $1(0.9)$ \\
\hline Acute transverse myelitis & $1(0.9)$ \\
\hline Viral myocarditis & $1(0.9)$ \\
\hline Toxic epidermal necrolysis (TEN) & $1(0.9)$ \\
\hline $\begin{array}{l}\text { Acute disseminated encephalomyelitis } \\
\text { (ADEM) }\end{array}$ & $1(0.9)$ \\
\hline Evan's syndrome & $1(0.9)$ \\
\hline Systemic lupus erythematous (SLE) & $1(0.9)$ \\
\hline
\end{tabular}


TABLE II - Utilization of IVIG by indication and evidence category

\begin{tabular}{lcc}
\hline Indication & Number of cases (\%) & Category evidence* \\
\hline Labeled use & & \\
Kawasaki disease & $31(27.0)$ & I \\
ITP & $24(20.9)$ & I \\
Primary immunodeficiency & $3(2.6)$ & I \\
CIDP & $2(1.7)$ & I \\
Acute GVHD & $1(0.9)$ & IIa \\
Off-labeled, potentially indicated & & \\
Neonatal sepsis & $31(27.0)$ & IIb \\
Severe NNJ secondary to ABO incompatibilities & & I \\
Guillain-Barré syndrome & $8(7.0)$ & I \\
Perinatal-acquired varicella zoster infection (prophylaxis) & $2(1.7)$ & IIb \\
Neonatal autoimmune thrombocytopenia & $2(1.7)$ & IIb \\
Anti-NMDA receptor encephalitis & $2(1.7)$ & III \\
TEN & $1(0.9)$ & IIb \\
ADEM & $1(0.9)$ & IIa \\
Evan's syndrome & $1(0.9)$ & IIb \\
Off-labeled, not indicated & & \\
SLE & $1(0.9)$ & III \\
Multiple sclerosis & $1(0.9)$ & IIb \\
Varicella pneumonitis & $1(0.9)$ & III \\
Acute transverse myelitis & $1(0.9)$ & III \\
Viral myocarditis & $1(0.9)$ & III \\
\hline Clasiffcan of evis & &
\end{tabular}

* Classification of evidence levels (Selin, 2002):

- Category I: Medical conditions for which there is convincing evidence of benefit resulting from IVIG therapy.

- Category II: Medical conditions for which there is inconclusive evidence of benefit from IVIG therapy, because of either conflicting evidence, low level evidence, or limited research, possibly because the condition is rare. Category II is subdivided into "a" (high) and "b" (low), depending on whether there is a higher or lower level of evidence of benefit.

- Category III: Medical conditions for which there is convincing evidence that IVIG has no benefit, or for which there is no convincing evidence of benefit of IVIG therapy.

\section{DISCUSSION}

In our study, nearly half of the IVIG treatment given through the years 2007-2014 were for off-labeled indications. This value is much higher than those reported in the other studies. A study conducted in Singapore over a 10-year period in two public pediatric hospitals reported that IVIG was used for off-labeled indications in only less than $25 \%$ of cases (Wu et al., 2013). A study on IVIG use in the 10 institutions in Canada from 1997 to 1999 found that $38 \%$ of children received IVIG for offlabeled indications (Hanna et al., 2003). Another report from Spain on the utilization of IVIG from 2000 to 2004 that included 273 patients found that only $14 \%$ of IVIG courses were prescribed for off-labeled indications (Badia, Cardona, Massanes, 2006). The widespread use of IVIG for off-labeled indications, emergence of new therapeutic indications, and indefinite duration of use in neurological illnesses in addition to immune deficiencies have been linked with the shortage of IVIG supply (Provan et al., 2007).

In this study, the utilization of IVIG in the adjunctive treatment of neonatal sepsis and severe neonatal jaundice secondary to $\mathrm{ABO}$ incompatibility contributed to the high usage of IVIG for off-labeled indications in the study. A meta-analysis of 110 cases of neonatal sepsis administered with IVIG found a 6-fold reduction in mortality compared to controls (Jenson, Pollock, 1997). Another meta-analysis 
TABLE III - Association between demographics and clinical data with labeled and off-labeled indications

\begin{tabular}{|c|c|c|c|}
\hline Parameter & Labeled $(\mathrm{n}=61)$ & Off-labeled $(\mathrm{n}=54)$ & p-value \\
\hline Age, years [median (IQR)] & $1.25(0.67-2.42)$ & $0.02(0.01-0.17)$ & $<0.001^{\mathrm{b}}$ \\
\hline Gender, $n(\%)$ & & & $0.857^{\mathrm{a}}$ \\
\hline Male & $34(55.7)$ & $31(57.4)$ & \\
\hline Female & $27(44.3)$ & $23(42.6)$ & \\
\hline Ethnicity, $n(\%)$ & & & $0.335^{\mathrm{c}}$ \\
\hline Malay & $39(63.9)$ & $36(66.7)$ & \\
\hline Chinese & $20(32.8)$ & $13(24.1)$ & \\
\hline Indian & $2(3.3)$ & $2(3.7)$ & \\
\hline Others & 0 & $3(5.6)$ & \\
\hline Body weight, kg [median (IQR)] & $9.10(7.12-11.80)$ & $2.36(0.96-3.28)$ & $<0.001^{\mathrm{b}}$ \\
\hline Specialty, n (\%) & & & $<0.001^{\mathrm{c}}$ \\
\hline Neurology & $2(3.3)$ & $5(9.3)$ & \\
\hline Hematology & $31(50.8)$ & - & \\
\hline Immunology & $3(4.9)$ & $1(1.9)$ & \\
\hline Dermatology & $24(39.3)$ & $1(1.9)$ & \\
\hline Neonatology & - & $44(81.5)$ & \\
\hline Others & $1(1.6)$ & $3(5.6)$ & \\
\hline Total usage, g [median (IQR)] & $14.00(9.00-20.00)$ & $2.50(1.30-3.00)$ & $<0.001^{\mathrm{b}}$ \\
\hline Length of hospital stay (day) & $5.00(3.00-8.00)$ & $15.50(5.00-53.00)$ & $<0.001^{\mathrm{b}}$ \\
\hline All cause mortality, $n(\%)$ & & & $<0.001^{\mathrm{c}}$ \\
\hline Yes & $1(1.6)$ & $15(27.8)$ & \\
\hline No & $60(98.4)$ & $39(72.2)$ & \\
\hline Adverse reaction, $n(\%)$ & & & $0.705^{\mathrm{c}}$ \\
\hline Yes & $3(4.9)$ & $4(7.4)$ & \\
\hline No & $58(95.1)$ & $50(92.6)$ & \\
\hline
\end{tabular}

$\mathrm{a}=$ Chi Square test; $\mathrm{b}=$ Mann-Whitney U test, $\mathrm{c}=$ Fisher's Exact test. ${ }^{*} \mathrm{p}<0.05$ denoted statistical significant difference. $\mathrm{IQR}=$ interquartile range

on the assessment of its effectiveness found a significant reduction in mortality in infants with suspected or proven infection; however, a concern about the small sample sizes and study quality was raised (Ohlsson, Lacy, 2015). In 2011, the International Neonatal Immunotherapy Study (INIS) Collaborative Group published a placebo-controlled trial on 3493 low-weight infants and found no significant difference in the rate of death or major disability at the age of 2 years between patients receiving placebo or those under IVIG treatment for neonatal sepsis (Brocklehurst et al., 2011). Based on the evidence from this latest prospective, randomized and placebo-controlled trial, the United Kingdom clinical guideline on IVIG use had listed neonatal sepsis as one of the "not recommended indication" for IVIG therapy with grade A recommendation and level Ia evidence level (Provan et al., 2011).
Few studies on neonatal jaundice found the beneficial effect of IVIG administration in neonates with isoimmune hemolytic jaundice. Two systematic reviews demonstrated that IVIG significantly reduced the need for exchange transfusion in neonates with hemolytic disease of the fetus and newborn (Alcock, Liley, 2002; Gottstein, Cooke, 2003). Moreover, exchange transfusion was reported to be associated with morbidity and mortality (Anderson et al., 2007).

For all labeled indications, the recommended IVIG dose and frequency are according to the guidelines of the British Columbia IVIG Utilization Management Handbook and the Canadian Blood Service 2000 national consensus conference on IVIG. For off-labeled indications, IVIG was administered using a variety of dosing regimens but still following the usual recommended dose in labeled 
indications, which ranged from $0.4 \mathrm{~g} / \mathrm{kg}$ to $2.0 \mathrm{~g} / \mathrm{kg}$ per course in either a single dose or few divided doses (Prasad, Chaudhary, 2014).

The incidence of adverse reaction in this study was $6.1 \%$, which falls in the range of $3-15 \%$ as reported in the literature (Goddard, 2008). The Naranjo scale, an adverse drug reaction probability scale, was used to assess the causality of IVIG-related adverse reaction in 7 reported cases in this study. All the 7 cases ( 5 mild cases, 1 moderate case, and 1 severe case) were interpreted as a probable level of IVIG infusion-related adverse reaction. The probable level of the Naranjo score is defined as the adverse reaction that occurred following a reasonable temporal sequence after drug administration and a recognized response to the suspected drug. It was confirmed by withdrawal but not by exposure to the drug and could not be reasonably explained by the known characteristics of the patient's clinical state (Naranjo et al., 1981).

In the present study, the indication of IVIG as labeled or off-labeled was found to be associated with the patient's age, body weight, specialty, total IVIG usage, length of hospital stay, and all-cause mortality rate. Neonatal sepsis and severe NNJ secondary to $\mathrm{ABO}$ incompatibility were the most common off-label indications for IVIG administration. Hence, the lower total usage of IVIG for off-labeled indications compared to the labeled indications could be explained by the lower body weight of neonates. Other various factors such as underlying illness may contribute to the longer hospital stay and higher mortality rate seen in patients receiving IVIG for off-label indications.

In view of the high usage of IVIG for off-label indications, a strict approval system for IVIG prescription is needed. In many institutions, policies have been developed to monitor and control the dispensing process of IVIG. In a Spanish institution, the pharmacists assess the indication and categorize it upon receipt of prescription for IVIG. If the IVIG is requested for off-labeled indications, the pharmacist will contact the prescriber to obtain the documentation required to request authorization from the health authorities for compassionate use (Badia, Cardona, Massanes, 2006). A study conducted in Saudi Arabia had showed a clear improvement in the ratio of labeled to offlabeled use and correct dosing for various indications after the adoption of an IVIG indication form. Any off-labeled indication must obtain approval from the clinical research committee (Frayha et al., 1997). Hence, all these strategies could be implemented for optimal IVIG utilization in the present setting.

There were several limitations of this study. First, there were medical records that could not be traced from the years 2007 to 2010 . For that reason, the exact usage of IVIG could be higher than that reported in this study. Second, the length of hospital stays and mortality rate could be affected by other comorbidities or coinfection present and not just due to the IVIG treatment.

\section{CONCLUSION}

IVIG therapy used in this setting for off-labeled indications was high with indications that may not benefit from the IVIG therapy due to the lack of strong clinical evidence. The patient's age, body weight, specialty, total IVIG usage, length of hospital stays, and all-cause mortality rate were found to be associated with IVIG indications being labeled or off-labeled, although there are other confounding factors that may have influenced the results seen. A national policy on the prescription of IVIG needs to be urgently developed to guide physicians in appropriately prescribing IVIG for pediatric patients.

\section{ACKNOWLEDGEMENT}

The authors would like to thank the hospital medical records staff for their help in retrieving the medical records. There was no funding received for this research. The authors report no conflicts of interest in this work.

\section{REFERENCES}

Alcock GS, Liley H. Immunoglobulin infusion for isoimmune haemolytic jaundice in neonates. Cochrane Database Syst Rev. 2002;3:CD003313.

Anderson D, Ali K, Blanchette V, Brouwers M, Couban S, Radmoor P, et al. Guidelines on the use of intravenous immune globulin for hematologic conditions. Transfus Med Rev. 2007;21(2 Suppl 1):S9-56.

Badia MP, Cardona LP, Massanes RJ. Changes in the pattern of intravenous immunoglobulin use over a 5-year period. Eur J Hosp Pharm Sci Pract. 2006;12:67-71.

Bichuetti-Silva DC, Furlan FP, Nobre FA, Pereira CT, Gonçalves TR, Gouveia-Pereira M, et al. Immediate infusionrelated adverse reactions to intravenous immunoglobulin in a prospective cohort of 1765 infusions. Int Immunopharmacol. 2014;23(2):442-6. 
Brennan VM, Salomé-Bentley NJ, Chapel HM. Prospective audit of adverse reactions occurring in 459 primary antibodydeficient patients receiving intravenous immunoglobulin. Clin Exp Immunol. 2003;133(2):247-51.

Brocklehurst P, Farrell B, King A, Juszczak E, Darlow B, Haque K, et al. Inis Collaborative Group. Treatment of neonatal sepsis with intravenous immune globulin. N Engl J Med. 2011;365(13):1201-11.

Frayha HH, Nuessle SJ, Arishi H, Rayes H, Qunibi WY, Bazarbashi MS. Improving utilization of intravenous immune globulin through concurrent use of an indication form. Eur J Clin Pharmacol. 1997;52(4):255-60.

Goddard EA. Intravenous immunoglobulin. Curr Allergy Clin Immunol. 2008;21(1):26-31.

Gottstein R, Cooke RW. Systematic review of intravenous immunoglobulin in haemolytic disease of the newborn. Arch Dis Child Fetal Neonatal Ed. 2003;88(1):F6-10.

Hanna K, Poulin-Costello M, Preston M, Maresky N. Intravenous immune globulin use in Canada. Can J Clin Pharmacol. 2003;10(1):11-6.

Jenson HB, Pollock BH. Meta-analyses of the effectiveness of intravenous immune globulin for prevention and treatment of neonatal sepsis. Pediatrics. 1997;99(2):E2.

Leong H, Stachnik J, Bonk ME, Matuszewski KA. Unlabeled uses of intravenous immune globulin. Am J Health Syst Pharm. 2008;65(19):1815-24.

Naranjo CA, Busto U, Sellers EM, Sandor P, Ruiz I, Roberts EA, et al. A method for estimating the probability of adverse drug reactions. Clin Pharmacol Ther. 1981;30(2):239-45.

Ohlsson A, Lacy JB. Intravenous immunoglobulin for suspected or subsequently proven infection in neonates. Cochrane Database Syst Rev. 2015;27(3):CD001239.
Orange JS, Hossny EM, Weiler CR, Ballow M, Berger M, Bonilla FA, et al. Use of intravenous immunoglobulin in human disease: a review of evidence by members of the Primary Immunodeficiency Committee of the American Academy of Allergy, Asthma and Immunology. J Allergy Clin Immunol. 2006;117(4 Suppl):S525-53.

Prasad AN, Chaudhary S. Intravenous immunoglobulin in pediatrics: a review. Med J Armed Forces India. 2014;70(3):27780 .

Provan D, Nokes T, Agrawal S, Winer J, Wood P. Clinical guidelines for the use of intravenous immunoglobulin. $2^{\text {nd }} e d$. update. London: Department of Health; 2011.

Provan D, Nokes T, Agrawal S, Winer J, Wood P. Clinical guidelines for the use of intravenous immunoglobulin. London: Department of Health; 2007.

Selin S. IVIG utilization in British Columbia. IVIG utilization management handbook. Vancouver: Provincial Blood Coordinating Office; 2002, p.4-6.

Sher G, Chiavetta G, Newman A, Gula C, Boyce C. Summary of national data on Canadian IVIG usage. Canadian Blood Services Conference; 2000.

Stiehm ER, Keller MA, Vyas GN. Preparation and use of therapeutic antibodies primarily of human origin. Biologicals. 2008;36(6):363-74.

Wu J, Lee AJ, Goh AE, Chia M, Ho C, Bugarin JL, et al. Use of intravenous immunoglobulin in an Asian paediatric population over a 10-year period. J Paediatr Child Health. 2013;49(8):62934.

Received for publication on $12^{\text {th }}$ October 2016 Accepted for publication on $14^{\text {th }}$ September 2017 